\title{
Finite-element modeling of spontaneous emission of a quantum emitter at nanoscale
} proximity to plasmonic waveguides

Chen, Yuntian; Nielsen, Torben Roland; Gregersen, Niels; Lodahl, Peter; Mørk, Jesper

Published in:

Physical Review B Condensed Matter

Link to article, DOI:

10.1103/PhysRevB.81.125431

Publication date:

2010

Document Version

Publisher's PDF, also known as Version of record

Link back to DTU Orbit

Citation (APA):

Chen, Y., Nielsen, T. R., Gregersen, N., Lodahl, P., \& Mørk, J. (2010). Finite-element modeling of spontaneous emission of a quantum emitter at nanoscale proximity to plasmonic waveguides. Physical Review $B$ Condensed Matter, 81(12), 125431. https://doi.org/10.1103/PhysRevB.81.125431

\section{General rights}

Copyright and moral rights for the publications made accessible in the public portal are retained by the authors and/or other copyright owners and it is a condition of accessing publications that users recognise and abide by the legal requirements associated with these rights.

- Users may download and print one copy of any publication from the public portal for the purpose of private study or research.

- You may not further distribute the material or use it for any profit-making activity or commercial gain

- You may freely distribute the URL identifying the publication in the public portal 


\title{
Finite-element modeling of spontaneous emission of a quantum emitter at nanoscale proximity to plasmonic waveguides
}

\author{
Yuntian Chen, ${ }^{*}$ Torben Roland Nielsen, Niels Gregersen, Peter Lodahl, and Jesper Mørk \\ DTU Fotonik, Department of Photonics Engineering, Ørsteds Plads, Building 345v, DK-2800 Kongens Lyngby, Denmark
}

(Received 25 August 2009; published 25 March 2010)

\begin{abstract}
We develop a self-consistent finite-element method to quantitatively study spontaneous emission from emitters in nanoscale proximity of plasmonic waveguides. In the model, it is assumed that only one guided mode is dominatingly excited by the quantum emitter, while the cross section of the plasmonic waveguide can be arbitrary. The fraction of the energy coupled to the plasmonic mode can be calculated exactly, which can be used to determine the efficiency with which single optical plasmons are generated. We apply our numerical method to calculate the coupling of a quantum emitter to a cylindrical metallic nanowire and a square metallic waveguide, and compare the cylindrical metallic nanowire with previous work that employs quasistatic approximation. For the cylindrical metallic nanowire we observe good agreement with the quasistatic approximation for radii below $10 \mathrm{~nm}$, but for increasing radius the spontaneous emission $\beta$ factor and the plasmonic decay rate deviate substantially, by factors of up to $5-10$ for a radius of $\sim 100 \mathrm{~nm}$, from the values obtained in the quasistatic approximation. We also show that the quasistatic approximation is typically valid when the radius is less than the skin depth of the metals at optical frequencies. For the square metallic waveguide we estimate an optimized value for the spontaneous emission $\beta$ factor up to $80 \%$.
\end{abstract}

DOI: 10.1103/PhysRevB.81.125431

\section{INTRODUCTION}

It has long been realized that the spontaneous emission rate is not an intrinsic property of a quantum emitter itself. ${ }^{1}$ The general explanation is that the spontaneous emission rate depends on the transition strength between the upper and lower levels of the quantum emitter as well as the local density of optical states. The local density of states (LDOS) measures the available number of electromagnetic modes into which the photons can be emitted at a specific location of the emitter and can be manipulated by tailoring the photonic environment of the emitter. A number of structures such as interfaces, ${ }^{2,3}$ cavities, ${ }^{4,5}$ photonic crystals, ${ }^{6,7}$ and waveguides ${ }^{8,9}$ have already been used to modify the spontaneous emission rate. Apart from fundamental studies, engineering the spontaneous emission rate of a quantum emitter can lead to new possibilities to boost the efficiency of optoelectronic devices, i.e., single-photon sources, low threshold lasers, and LED lightening.

As an alternative to dielectric materials, the spontaneous emission rate can be manipulated by subwavelength metallic systems, which support surface plasmon polaritons. Surface plasmon polaritons are electromagnetic excitations associated with charge density waves on the surface of a conducting object. The tight confinement of the electromagnetic field to the metal-dielectric interface due to the boundary condition constraints gives the possibility of inventing new ways to enhance light-matter interaction, such as efficient single optical plasmon generation, ${ }^{10,11}$ single molecule detection with surface-enhanced Raman scattering, ${ }^{12,13}$ enhanced photoluminescence from quantum wells, ${ }^{14}$ and nanoantenna modified spontaneous emission. ${ }^{15-17}$

Although limited by the intrinsic losses of the metals in the optical frequency range, different metallic structures have been extensively studied in the last few years due to the possibilities of integration and miniaturization. The dramatic enhancement of the field intensity due to the field concentra-
PACS number(s): 73.20.Mf, 78.55.-m, 42.50.Pq, 03.67.-a

tion and geometric slowing down of the mode propagation provides an excellent platform to study single-photon nonlinear optics ${ }^{18}$ and light-matter interaction at the single-emittersingle-photon level. There are also considerable interests in surface plasmons for subwavelength optics ${ }^{19}$ and applications in sensing, near-field imaging, waveguiding, and switching below the diffraction limit. ${ }^{20-23}$ The study of plasmonic effects to enhance light-matter interaction and the preferential spontaneous emission from, e.g., a quantum dot into a desired mode is currently a hot research topic. It is important for solid-state quantum information devices as well as for improving our understanding of light-matter interaction at the nanoscale. So far, there are only a few theoretical papers ${ }^{11,24}$ on this topic, and they employ simplifying assumptions that limit their applicability for analyzing realistic structures, e.g., by assuming geometrical shapes that are not readily achievable using current fabrication technology and making assumptions that are only valid at some length scales. The realistic description of all competing, radiative and nonradiative, decay channels for an emitter placed in close proximity to a plasmonic waveguide of general geometry is important in order to understand the physics and the fundamental limitations.

The present paper focuses on modeling of the spontaneous emission of a quantum emitter at nanoscale proximity to realistic plasmonic waveguides by using a finite-element method (FEM) with special emphasis on calculating the spontaneous emission $\beta$ factor. The $\beta$ factor describes the fraction of the emitted energy that is coupled to the plasmonic mode. Subwavelength waveguiding of plasmons in metallic structures has been studied theoretically ${ }^{22,25}$ and has also been observed in a number of recent experiments. ${ }^{23}$ Enhanced spontaneous emission of an emitter coupled to plasmonic waveguides has been proposed ${ }^{11,26}$ and experimentally demonstrated ${ }^{10}$ recently. Chang et al. ${ }^{24}$ studied the spontaneous emission of an emitter coupled to a metallic nanowire by exploiting, except for a single example, the quasistatic ap- 
proximation. Their model qualitatively shows that, for small nanowire radii, it is possible to obtain efficient coupling without taking into account the wave properties of the plasmonic mode. Jun et al. ${ }^{26}$ employed an finite-difference timedomain (FDTD) numerical method to study the different spontaneous emission decay rates of an emitter coupled to a metallic slot waveguide, but using simplifying assumptions for the LDOS calculations of the plasmonic mode. A selfconsistent model with rigorous treatment of all the spontaneous decay rates involved, i.e., radiative as well as nonradiative, has not been presented in the literature. The aim of this paper is to provide such a detailed modeling and to extend earlier results to realistic structures.

This paper is organized as follows. In Sec. II, the computational principle and the numerical method are presented. First we study the dispersion relation and the mode properties of the plasmonic waveguide, and then we calculate the decay rate into the plasmonic channel in a two-dimensional (2D) model by taking advantage of the translational symmetry of the waveguides. Finally, the wave equation with a current source is solved numerically for a full threedimensional (3D) configuration, and the total decay rate of the quantum emitter is extracted by calculating the total power emitted from the current source. Section III presents results obtained by applying the numerical method to two different plasmonic waveguides. The results for the cylindrical nanowire are compared to those obtained in the quasistatic approximation. We observe substantial differences and give a qualitative assessment regarding the validity of the quasistatic approximation. As an inspiration for many other geometries, which are particularly interesting when considering available fabrication technology, the square plasmonic waveguide is also studied. Section IV concludes the paper.

\section{COMPUTATIONAL APPROACH}

As shown in Fig. 1, we consider an ideal quantum emitter coupled to a plasmonic waveguide. The excitation energy of the quantum emitter can be dissipated either radiatively or nonradiatively. Radiative relaxation is associated with the emission of a photon, whereas nonradiative relaxation can be various pathways such as coupling to vibrations, resistive heating of the environment, or quenching by other quantum emitters. The resistive heating of the metallic waveguide is the only mechanism of nonradiative relaxation considered in our model. The quantum emitter is positioned in the vicinity of the metallic nanowire, thus there are three channels for the quantum emitter to decay into, i.e., the radiative channel, the plasmonic channel, and the nonradiative channel. The corresponding decay rates are denoted by $\gamma_{\text {rad }}, \gamma_{p l}$, and $\gamma_{\text {nonrad }}$, respectively. The radiative channel accounts for the spontaneous emission in the form of far field radiation. The plasmonic channel is the excitation of the plasmonic mode, which is guided by the plasmonic waveguide. The nonradiative channel is associated with the resistive heating of the lossy metals, which is due to electron-hole pair generation inside the metals. The spontaneous emission $\beta$ factor is defined by $\beta=\frac{\gamma_{p l}}{\gamma_{\text {total }}}$, where $\gamma_{\text {total }}$ is the sum of the three rates, $\gamma_{\text {total }}=\gamma_{\text {rad }}+\gamma_{\text {nonrad }}+\gamma_{p l}$. The $\beta$ factor gives the probability

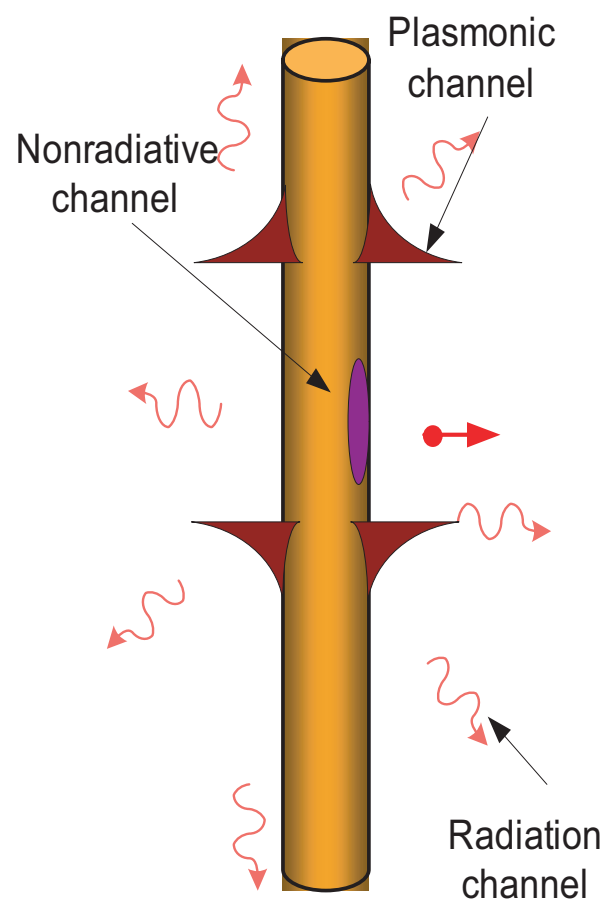

FIG. 1. (Color) Different emission channels involved in the decay process of a quantum emitter (red dot) coupled to a plasmonic waveguide. In the radiation channel the photons are traveling in free space. In the plasmonic channel the plasmonic modes are excited and guided by the metallic nanowire. In the nonradiative channel, electron-hole pairs are generated.

that the quantum emitter excites a single plasmonic mode.

\section{A. Dispersion relation and decay rate into the plasmonic channel}

The starting point of the numerical analysis of the waveguide is the wave equation for the electric field,

$$
\nabla \times\left(\frac{\nabla \times \bar{E}(\bar{r})}{\mu_{r}}\right)-k_{0}^{2} \varepsilon(\bar{r}) \bar{E}(\bar{r})=0,
$$

where $k_{0}=\omega \sqrt{\varepsilon_{0} \mu_{0}}$ is the vacuum wave number, $\varepsilon(\vec{r})$ denotes the dielectric function relative to vacuum, and $\mu_{r}$ represents the relative permeability constant, which is assumed to be 1 throughout the paper. Due to the invariance along the $Z$ axis, the $Z$ dependence of the solution to the wave equation must be that of a plane wave (complex exponential),

$$
\bar{E}(x, y, z)=\bar{E}_{\alpha}(x, y) e^{-j(\omega t-\beta z)} .
$$

For the guided plasmonic modes, at a specific frequency $\omega$, two quantization indices are needed to specify a complete set of orthogonal modes, i.e., $\alpha=\{p, \beta\}$. $\beta$ denotes the propagation constant (the component of the wave vector along the $Z$ axis), and the index $p$ represents the polarization of the mode. The waveguide structure examined consists of two regions $\Omega$ and $\Lambda$. $\Omega$ is the lossy metal core, which is surrounded by an infinite lossless dielectric medium $\Lambda$. The transverse component of the wave vector, defined as $k_{i \perp}$ $=j \kappa_{i \perp}$, fulfills $j \kappa_{i \perp}=\sqrt{\frac{\omega^{2}}{c^{2}} \varepsilon_{i}-\beta^{2}}$ with $i \in[\Omega, \Lambda]$, where $\varepsilon_{i}$ is the relative permittivity. 


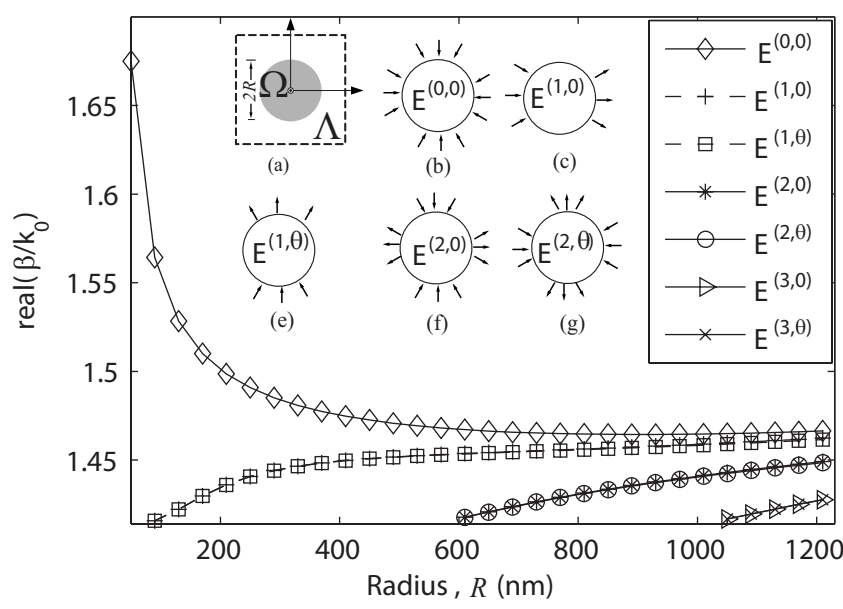

FIG. 2. Dispersion relation versus radius for the cylindrical gold nanowire with the background medium of PMMA $(n=1.414)$ at the wavelength of $1 \mu \mathrm{m}$. Inset (a) shows the waveguide structure. Inset (b)-(g) show electric field orientation of the possible eigenmodes supported by the waveguide.

The FEM method is utilized as a numerical tool to calculate the guided plasmonic modes, the reason for choosing this method will be discussed later. The infinite dielectric medium is truncated to perform the finite-element analysis of the waveguide structure by placing the structure inside a computational window, which is large enough to guarantee the field vanishing at the boundary. Here, we consider an optical wavelength of $1 \mu \mathrm{m}$ and the relative optical permittivities of the waveguide are $\varepsilon_{\Omega}=-50+3.85 i$ and $\varepsilon_{\Lambda}=2$, corresponding to gold ${ }^{27}$ and an ordinary polymer, i.e., polymethylmethacrylate (PMMA). The dispersion and the field orientation of the possible modes for cylindrical and square waveguides are presented in Figs. 2 and 3, respectively. As shown in the inset of Fig. 2, these modes can be represented by two indices, where the first index corresponds to the mode with angular moment of $m$ and the second index describes the polarization degenerate mode with the same $m$. For ex-

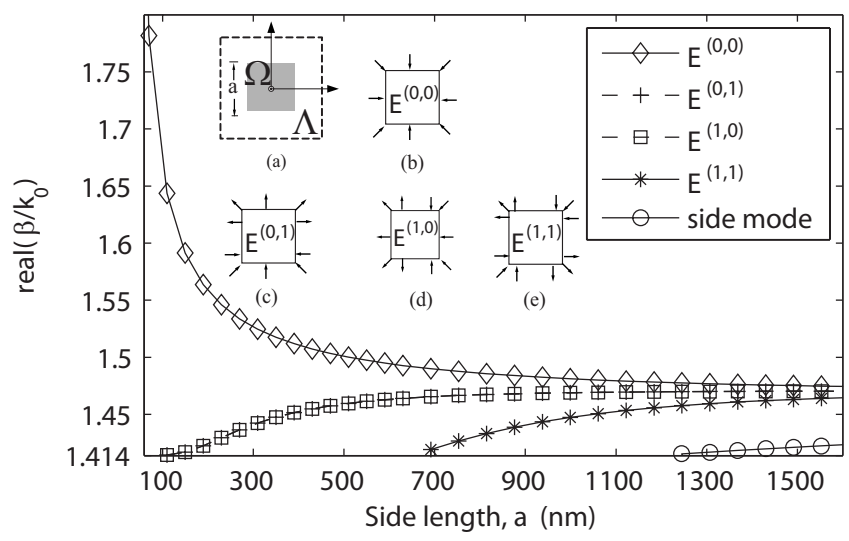

FIG. 3. Dispersion relation versus side length of square plasmonic waveguides with background medium of PMMA ( $n$ $=1.414$ ) at a wavelength of $1 \mu \mathrm{m}$. The metal core is gold. Inset (a) shows the waveguide structure. Inset (b)-(e) show electric field orientation of the possible eigenmodes supported by square plasmonic waveguides. ample, if $E^{m, 0}$ denotes a mode related with angular moment of $m$, then $E^{m, \theta}$ denotes the corresponding degenerated mode, the field distribution of which is rotated by $\theta$ along the $Z$ axis compared with $E^{m, 0}$, where $\theta=\pi / 2 m$. As pointed out by Takahara et al. ${ }^{22}$ the fundamental mode $E^{(0,0)}$ does not have a cut-off size of the radius, which is confirmed by the dispersion relation in Fig. 2. The modes supported by the metallic nanowire preserve the cylindrical symmetry of the waveguide. Due to the constraints from the boundary condition, only TM modes exist. For the square surface plasmon polariton waveguides, the fundamental modes, which were studied by Jung et al. ${ }^{28}$ can be labeled in terms of two indices, which denote the number of sign changes in the dominant component of the electric field along the $X$ and $Y$ axes, respectively. Both plasmonic waveguides support one fundamental mode $\left(E^{0,0}\right)$ without any cut-off size of the metal core, and the corresponding propagation constants increase when the size of the metal core is further shrunk, which slows down the propagating plasmonic mode. Such geometric slowing down enhances the LDOS and the coupling efficiency to a nearby quantum emitter. We note that plasmonic modes supported by the metallic strips attracted considerable interest recently, due to the tight confinement of the field as well as the long-range propagation. ${ }^{29-32}$ In the following calculations, the size of the metal core is restricted to be below the cut-off size of higher order modes so that only a single mode is supported. We note that apart from the highly localized mode, a weakly localized mode also exists. However, since the contribution to the total decay rate from such a weakly guided mode is small, we simply treat this mode as one of the radiation modes in our model.

The electric field dyadic Green's function for a specific guided plasmonic mode is constructed from the numerical calculation of the electric field. In the following part we will explain how to construct the electric field dyadic Green's function for one guided plasmonic mode. ${ }^{33}$

The electric dyadic Green's function $\overline{\bar{G}}\left(\bar{r}, \bar{r}^{\prime}, \omega\right)$ is defined by

$$
\left[\nabla \times \nabla \times-k_{0}^{2} \varepsilon(\bar{r})\right] \overline{\bar{G}}\left(\bar{r}, \bar{r}^{\prime}, \omega\right)=\overline{\bar{I}} \delta\left(\bar{r}-\bar{r}^{\prime}\right),
$$

where $\overline{\bar{I}}$ is the unit dyad. Rigorously speaking, the operator defined by $\hat{L}=\left[\nabla \times \nabla \times-k_{0}^{2} \varepsilon(\vec{r})\right]$ does not have a set of complete and orthogonal eigenmodes due to its non-Hermitian character if $\varepsilon(\vec{r})$ is complex. Without loss of generality, we adopt biorthogonality in the present paper to form a complete set of "orthogonal" modes of the waveguides initially, and then we will end up with an approximation from the power orthogonality for the plasmonic waveguides. Suppose that $\bar{E}_{n}$ is a set of eigensolutions defined by $\hat{L}$, the biorthogonal modes $\bar{E}_{m}^{\dagger}$ are defined as the eigensolutions of the adjoint operator denoted by $\hat{L}^{\dagger}$, which is obtained from the operator $\hat{L}$ by replacing $\varepsilon(\vec{r})$ with its complex conjugate. The biorthogonality condition is then given by

$$
\int \varepsilon(\vec{r}) \bar{E}_{n}(\bar{r}) \cdot\left[\bar{E}_{m}^{\dagger}(\vec{r})\right]^{*} d^{3} r=\delta_{n m} N_{n},
$$

with the completeness relation $\Sigma_{n} \frac{\varepsilon(\vec{r}) \bar{E}_{n}(\vec{r})\left[\bar{E}_{n}^{\dagger}\left(\bar{r}^{\prime}\right)\right]^{*}}{N_{n}}=\overline{\bar{I}} \delta\left(\bar{r}-\bar{r}^{\prime}\right)$. From the biorthogonal completeness relation, the dyadic 
Green's function $\overline{\bar{G}}\left(\bar{r}, \bar{r}^{\prime}, \omega\right)$ can be constructed from the eigenfunction expansion as follows: ${ }^{33}$

$$
\begin{aligned}
\overline{\bar{G}}\left(\bar{r}, \bar{r}^{\prime} \omega\right)= & \overline{\bar{G}}_{G L}\left(\bar{r}, \bar{r}^{\prime} \omega\right)+\overline{\bar{G}}_{G L}\left(\bar{r}, \bar{r}^{\prime} \omega\right)=\sum_{n} \frac{\bar{E}_{n}(\bar{r})\left[\bar{E}_{n}^{\dagger}\left(\bar{r}^{\prime}\right)\right]^{*}}{N_{n} \lambda_{n}} \\
& +\sum_{n} \frac{\nabla \phi_{n}(\bar{r})\left[\nabla \phi_{n}^{\dagger}\left(\bar{r}^{\prime}\right)\right]^{*}}{M_{n} k_{0}^{2}}
\end{aligned}
$$

where the generalized transverse part of the dyadic Green's function, $\overline{\bar{G}}_{G T}$, is constructed from the complete set of transverse eigenfunction $\bar{E}_{n}(\bar{r})$ given by

$$
\begin{gathered}
-\nabla \times \nabla \times \bar{E}_{n}(\bar{r})+k_{0}^{2} \varepsilon(\bar{r}) \bar{E}_{n}(\bar{r})=\lambda_{n} \varepsilon(\bar{r}) \bar{E}_{n}(\bar{r}), \\
\nabla \cdot\left[\varepsilon(\bar{r}) \bar{E}_{n}(\bar{r})\right]=0
\end{gathered}
$$

with the eigenvalue $\lambda_{n}$. The longitudinal or quasistatic part $\overline{\bar{G}}_{G L}$ is constructed from longitudinal eigenfunctions that can be found from a complete set of scalar eigenmodes $\phi_{n}(\vec{r})$ satisfying

$$
\nabla \cdot\left[\varepsilon(\bar{r}) \nabla \phi_{n}(\vec{r})\right]=\sigma_{n} \phi_{n}(\vec{r})
$$

with the biorthogonality relation, $\int \varepsilon(\vec{r}) \nabla \phi_{n}(\vec{r}) \cdot\left[\nabla \phi_{n}^{\dagger}(\vec{r})\right]^{*} d^{3} r=\delta_{n m} M_{n}$. Since we are studying the guided plasmonic mode, which describes the field solution in the absence of electric charges $\left(\nabla \cdot\left[\varepsilon(\vec{r}) \bar{E}_{n}(\vec{r})\right]=0\right)$, the longitudinal component will vanish in the following calculations.

By applying the principle of constructing the electric field dyadic Green's function to the case of a plasmonic waveguide, we find the contribution to the dyadic Green's function from the plasmonic modes as

$$
\overline{\bar{G}}_{p l}\left(\bar{r}, \bar{r}^{\prime} \omega\right)=\sum_{p} \int_{-\infty}^{+\infty} \frac{\varepsilon_{\Lambda} \bar{E}_{\alpha}(x, y)\left[\bar{E}_{\alpha}^{\dagger}\left(x^{\prime}, y^{\prime}\right)\right]^{*} e^{j \beta\left(z-z^{\prime}\right)}}{\left[k_{0}^{2} \varepsilon_{\Lambda}-\left(\beta^{2}-\kappa_{\Lambda \perp}^{2}\right)\right] N} d \beta,
$$

where the normalization factor $N$ is given by

$$
\begin{aligned}
\delta\left(\beta-\beta^{\prime}\right) \delta_{p p^{\prime}} N=\int \varepsilon(\vec{r}) \overline{E_{\alpha}}(\bar{r}) \cdot\left[\bar{E}_{\alpha^{\prime}}^{\dagger}(\bar{r})\right]^{*} d^{3} r \\
\quad=2 \pi \delta\left(\beta-\beta^{\prime}\right) \delta_{p p^{\prime}} \int \varepsilon(x, y) \bar{E}_{\alpha}(x, y) \cdot\left[\bar{E}_{\alpha^{\prime}}^{\dagger}(x, y)\right]^{*} d x d y,
\end{aligned}
$$

which can be further simplified as

$$
N=2 \pi \int \varepsilon(x, y) \bar{E}_{\alpha}(x, y) \cdot\left[\bar{E}_{\alpha}^{\dagger}(x, y)\right]^{*} d x d y,
$$

if one realizes that $\alpha$ and $\alpha^{\prime}$ are required to denote the same mode in Eq. (8). For one plasmonic mode, the expression (8) is evaluated in closed form by the method of contour integration as the integrand decays to zero at infinity in the upper and the lower $\beta$ planes,

$$
\begin{aligned}
\overline{\bar{G}}_{p l}(\bar{r}, \bar{r}, \omega) & =j 2 \pi \frac{\varepsilon_{\Lambda} \bar{E}_{\alpha_{0}}(x, y)\left[\bar{E}_{\alpha_{0}}^{\dagger}(x, y)\right]^{*}}{\frac{d\left(k_{0}^{2} \varepsilon_{\Lambda}\right)}{d \beta} N} \\
& =\frac{j \pi c^{2} \bar{E}_{\alpha_{0}}(x, y)\left[\bar{E}_{\alpha_{0}}^{\dagger}(x, y)\right]^{*}}{\omega N v_{g}},
\end{aligned}
$$

where $v_{g}$ is the group velocity, defined by $v_{g}=d \omega / d \beta$. The corresponding projected LDOS for one plasmonic mode can be calculated from the dyadic Green's function according to Novotny, ${ }^{34} \rho_{\mu}\left(r_{0}, \omega_{0}\right)=6 \omega\left[\bar{n}_{\mu} \cdot \operatorname{Im}\left\{\overline{\bar{G}}\left(r_{0}, r_{0}, \omega_{0}\right)\right\} \cdot \bar{n}_{\mu}\right] /\left(\pi c^{2}\right)$, where $n_{\mu}$ is the unit vector of the dipole moment. If the dipole emitter is oriented along the $X$ axis, the projected LDOS for the plasmonic mode is given by $\rho_{p l}(\bar{r}, \omega)$ $=6\left|E_{\alpha, x}(x, y)\right|^{2} /\left(N v_{g}\right)$. The spontaneous emission decay rate into the plasmonic mode can be calculated by $\gamma_{p l}$ $=\frac{\pi \omega_{0}}{3 \hbar \varepsilon_{0}}|\mu|^{2} \rho_{p l}(\bar{r}, \omega)$. Normalized by the spontaneous emission decay rate in the vacuum, the emission enhancement due to the plasmonic excitation is

$$
\frac{\gamma_{p l}}{\gamma_{0}}=\frac{6 \pi^{2} c^{3} E_{\alpha_{0}, X}(x, y)\left[E_{\alpha_{0}, X}^{\dagger}(x, y)\right]^{*}}{\omega_{0}^{2} N v_{g}} .
$$

Equation (10) gives a general expression for the spontaneous-emission decay rate into a guided mode, supported by a lossy or lossless waveguide. In dielectric waveguides, losses are generally small, and the biorthogonal modes $\bar{E}_{m}^{\dagger}$ can to a good approximation be replaced by the orthogonal mode $\bar{E}_{m}$. Such an approximation is also valid for our plasmonic waveguide, where the imaginary part of the propagation constant for the fundamental mode is around $1 \%$ of the real part. According to Snyder, ${ }^{35}$ the group velocity can be calculated by $v_{g}=\int_{A_{\infty}}(\vec{E}$ $\left.\times \bar{H}^{*}\right) \cdot \bar{z} d A / \int_{A_{\infty}} \varepsilon_{0} \varepsilon(x, y)|\bar{E}(x, y)|^{2} d A$, where $A_{\infty}$ denotes integration over the transverse plane. By applying the power orthogonal approximation and plugging the explicit form of the group velocity into Eq. (10), we obtain the following expression for the plasmonic decay rate of the fundamental mode:

$$
\frac{\gamma_{p l}}{\gamma_{0}}=\frac{3 \pi c \varepsilon_{0} E_{\alpha_{0}, X}(x, y) E_{\alpha_{0}, X}^{*}(x, y)}{k_{0}^{2} \int_{A_{\infty}}\left(\bar{E} \times \bar{H}^{*}\right) \cdot \bar{z} d A} .
$$

\section{B. Total decay rate}

As described in the previous section, the well-defined field components in the transverse plane of the waveguide give the possibility of constructing the plasmonic part of the dyadic Green's function numerically. The reason is that the field is concentrated around the metallic core and decays to zero on the borders when the modeling domain is reasonably large. Hence, the perfect electric conductor boundary condition is implemented to truncate the $2 \mathrm{D}$ modeling domain. However, for the radiation modes, the field components in the transverse plane of the waveguide do not vanish no matter how large the modeling domain is. Hence, it is extremely 
difficult to construct the dyadic Green's function numerically for the radiation modes in a similar way as for the guided mode. Therefore, we implement a 3D model to include the radiation modes, as well as the nonradiative contributions, by solving the wave equation with a harmonic (time-dependent) source term,

$$
\left[\nabla \times \frac{1}{\mu_{r}} \nabla \times-k_{0}^{2} \varepsilon(\bar{r})\right] \bar{E}(\bar{r}, \omega)-j \omega \mu_{0} \bar{J}(\omega)=0 .
$$

Substantial efforts have been made to solve a more general form of Eq. (12), namely, with arbitrary time dependence. However, only for a few geometries such as spheres and cylinders, one is able to obtain analytical solutions, by the assistance of, e.g., Mie's theory ${ }^{36}$ and other modal descriptions. ${ }^{37}$ For many other geometries, one has to resort to numerical methods, like FDTD method, FEM, or other methods. Though FDTD can model dielectric structures reasonably well, i.e., photonic crystals, it has severe drawbacks for modeling plasmonic structures. First, the rectangular grids typically used in FDTD are not suitable for complex plasmonic structures due to the high localization of the field at the metal-dielectric interfaces, which requires much finer grid resolution than modeling of dielectric structures. The piecewise constant approximation of the fields within grids in FDTD poses difficulties in applying boundary condition at the metal-dielectric interfaces, which is crucial for modeling the plasmonic structures. Second, in FDTD and other timedomain methods the dielectric functions of the material need to be approximated by proper analytical expressions which give rise to considerable error in broadband calculations. Apart from these general drawbacks, the LDOS calculations present additional challenges for FDTD, due to difficulties in accurately transforming $\bar{J}^{*} \cdot \bar{E}$ from the time to frequency domain, as explained in Koenderink et al..$^{38-40}$ However, FEM does not have such challenges due to the more advanced discretization strategy for complex geometric structures by using a variety of elements of different shapes, and FEM is a frequency method which can handle arbitrary material dispersion. One might consider other options, i.e., the Green's dyadic function method ${ }^{41}$ or point dipole method, ${ }^{42}$ however, the Green's dyadic function method encounters difficulties in constructing Green's functions for complex geometries, and the point dipole model is limited to homogenous dielectric environments in which the metals are embedded. Since we want to develop a generally applicable quantitative method of modeling spontaneous emission for complex plasmonic structures, FEM is chosen as the numerical tool to attack the problem.

In this paper, we introduce the FEM method to calculate the LDOS in a 2D model and solve an equivalent problem in a 3D model. We found that the LDOS can be efficiently extracted from the FEM numerical calculations, without the numerical difficulties encountered in FDTD, when the boundary condition of the modeling domain is properly handled. Concerning implementation of a FEM calculation, Eq. (12) needs to be reformulated. If we introduce a test function $\bar{F}(\bar{r}, \omega)$, we can construct the functional corresponding to the wave equation in the following way: ${ }^{43}$

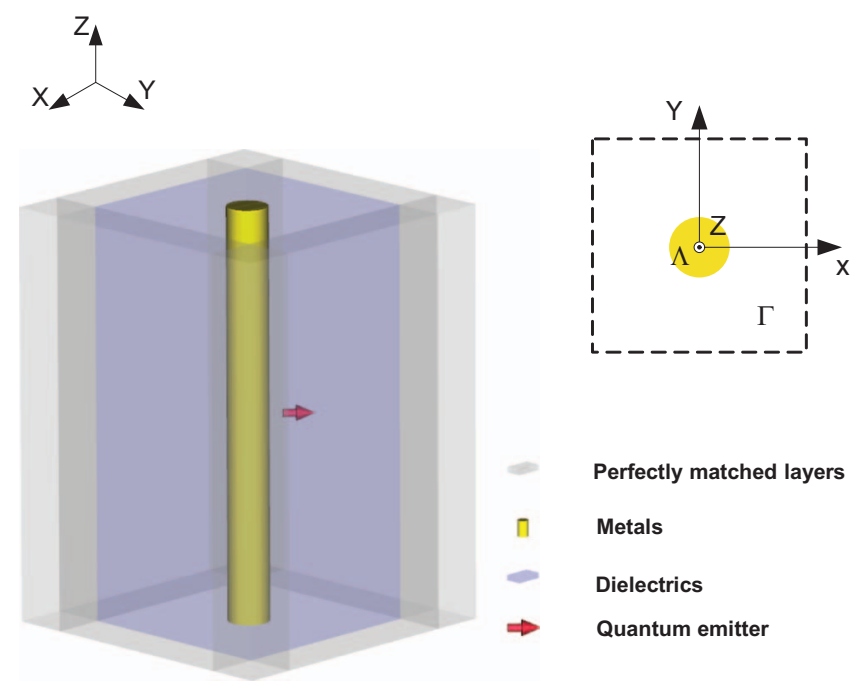

FIG. 4. (Color) A single quantum emitter coupled to a metallic nanowire. The gray transparent region represents the perfectly matched layers, the mode matching boundary condition is applied on the top and the bottom of the structure. The quantum emitter is implemented by an electric line current.

$$
\begin{aligned}
L= & \int_{V}\left[\nabla \times \frac{1}{\mu_{r}} \nabla \times-k_{0}^{2} \varepsilon(\bar{r})\right] \bar{E}(\bar{r}, \omega) \cdot \bar{F}^{*}(\bar{r}, \omega) d V \\
& -\int_{V} j \omega \mu_{0} \bar{J}(\omega) \cdot \bar{F}^{*}(\bar{r}, \omega) d V \\
= & \int_{V} \frac{1}{\mu_{r}} \nabla \times \bar{E}(\bar{r}, \omega) \cdot \nabla \times \bar{F}^{*}(\bar{r}, \omega) d V \\
& -\int_{V} k_{0}^{2} \varepsilon(\bar{r}) \bar{E}(\bar{r}, \omega) \cdot \bar{F}^{*}(\bar{r}, \omega) d V \\
& -\int_{V} j \omega \mu_{0} \bar{J}(\omega) \cdot \bar{F}^{*}(\bar{r}, \omega) d V \\
& +\oiint_{\partial V} \bar{F}^{*}(\bar{r}, \omega) \cdot\left[\frac{1}{\mu_{r}} \bar{n} \times \nabla \times \bar{E}(\bar{r}, \omega)\right] d s,
\end{aligned}
$$

where $\partial V$ denotes the surface that encloses the volume $V$ and $\bar{n}$ denotes the outward unit normal vector to the surface of the modeling domain. This is the variational formulation of the wave equation, which is required to hold for all test functions. Equation (13) enables us to formulate the finiteelement solution for such a boundary-value problem by employing the standard finite-element solution procedures, including discretization and factorization of a sparse matrix. ${ }^{43}$ The boundary-value problem defined by Eq. (13) was solved by utilizing a commercial software package, COMSOL MULTIPHYSICS. ${ }^{44}$

It is crucial to truncate the computational domain properly. As shown in Fig. 4, we use two techniques for truncating the modeling domain. (I) In the $X-Y$ plane, the computation domain is truncated by the perfectly matched layers with thickness of half a wavelength in vacuum. (II) Along the $Z$ axis, the computation domain is terminated by a mode 
TABLE I. The relations between the six field components for the fundamental hybrid mode.

\begin{tabular}{lc}
\hline \hline Description & Relation \\
\hline Tangential electric field, $s \in[x, y]$ & $E_{2 \mathrm{D}, t}^{s}=-\frac{\beta}{\omega \varepsilon_{0} \varepsilon(\bar{r})}\left(\bar{n} \times H_{2 \mathrm{D}, t}\right)_{s}-\frac{j}{\omega \varepsilon_{0} \varepsilon(\bar{r})}\left(\nabla_{t} \times \bar{n} H_{2 \mathrm{D}}^{n}\right)_{s}$ \\
Normal electric field & $E_{2 \mathrm{D}}^{n}=-\frac{j}{\omega \varepsilon_{0} \varepsilon(\bar{r})} \bar{n} \cdot\left(\nabla_{t} \times H_{2 \mathrm{D}, t}\right)$ \\
Tangential magnetic field, $s \in[x, y]$ & $H_{2 \mathrm{D}, t}^{s}=H_{2 d, m}^{s}$ \\
Normal magnetic field & $H_{2 \mathrm{D}}^{n}=j H_{2 d, m}^{z}$ \\
\hline
\end{tabular}

matching boundary condition, which will induce a certain amount of reflection from the radiation modes and the higher order plasmonic modes if they exist. Essentially, the mode matching boundary is an absorbing wall, which behaves as a sink of electromagnetic waves. There are different options for realizing the mode matching boundary to absorb a single mode, depending on whether the absorbed mode is TE, TM, or a hybrid mode. For a pure TM or TE mode, it can be matched by simply applying the conditions

$$
\begin{gathered}
\frac{1}{\mu_{r}} \bar{n} \times \nabla \times \bar{E}(\bar{r}, \omega)=-\frac{k_{0}^{2} \varepsilon(\bar{r}) \bar{E}_{t}(\bar{r}, \omega)}{j \beta}, \quad \mathrm{TM}, \\
\frac{1}{\mu_{r}} \bar{n} \times \nabla \times \bar{E}(\bar{r}, \omega)=-j \beta \bar{n} \times \frac{1}{\mu_{r}} \bar{n} \times \bar{E}_{t}(\bar{r}, \omega), \quad \text { TE, }
\end{gathered}
$$

on the boundary, where $\beta$ is the propagation constant, and $\bar{E}_{t}(\bar{r}, \omega)$ is the tangential components of the dependent variable $\bar{E}(\bar{r}, \omega)$ on the boundaries in the numerical model. The mode matching boundary condition for the hybrid mode can be implemented as

$$
\frac{1}{\mu_{r}} \bar{n} \times \nabla \times \bar{E}(\bar{r}, \omega)=j \omega \mu_{0} \bar{n} \times \bar{H}_{0},
$$

where $\bar{E}(\bar{r}, \omega)$ is the dependent variable solved in the $3 \mathrm{D}$ model and $\bar{H}_{0}$ denotes the matched mode that is applied. In our model, $\bar{H}_{0}$ corresponds to the fundamental hybrid mode supported by the plasmonic waveguide. It is calculated from the $2 \mathrm{D}$ eigenvalue problem and is given by

$$
\bar{H}_{0}=\sqrt{\frac{\gamma_{p l} P_{0}}{\gamma_{0} P_{2 d}}} \bar{H}_{2 d} e^{j \beta L_{0}}=\left(H_{0 x}, H_{0 y}, H_{0 z}\right) .
$$

Here, $P_{2 d}, \bar{H}_{2 d}$, and $\beta$ are the time-averaged power flow, the magnetic field, and the propagation constant, respectively, calculated from the 2D model, while $P_{0}$ denotes the normalization factor of the power emission in the $3 \mathrm{D}$ model, and $L_{0}$ represents the half length of the $3 \mathrm{D}$ model. Due to the losses of the metals, the magnitude of the magnetic field is a complex number. In order to guarantee that the phase of $E_{x}$ at the position of the emitter is zero when the emitter is oriented horizontally, the extra phase $\phi=\arctan \left(\frac{\operatorname{imag}\left(E_{2 \mathrm{D}}^{x}\right)}{\operatorname{real}\left(E_{2 \mathrm{D}}^{x}\right)}\right)$ needs to be compensated, i.e., $\bar{H}_{0}=\sqrt{\frac{\gamma_{p l} P_{0}}{\gamma_{0} P_{2 d}}} \bar{H}_{2 d} e^{j\left(\beta L_{0}-\phi\right)}$. In the 2D eigenvalue calculations, there are six components involved for the hybrid fundamental model, the relations of which are tabulated in Table I. The magnitudes of the magnetic field,
$\left(H_{2 d, m}^{x}, H_{2 d, m}^{y}, H_{2 d, m}^{z}\right)$, are the dependent variables, which are calculated directly from the 2D numerical model.

The total decay rate, $\gamma_{\text {total }}$, is extracted from the total power dissipation of the current source coupled to the nearby metallic waveguide, $\gamma_{\text {total }} / \gamma_{0}=P_{\text {total }} / P_{0}$, where $P_{\text {total }}$ $=1 / 2 \int_{V} \operatorname{Re}\left(\bar{J}^{*} \cdot \bar{E}_{\text {total }}\right) d V$ is the power dissipation of the current source coupled to the metallic waveguide and $P_{0}$ $=1 / 2 \int_{V} \operatorname{Re}\left(\bar{J}^{*} \cdot \bar{E}_{0}\right) d V$ is the emitted power by the same current source in vacuum. $P_{0}$ is a normalization factor, which is also used to normalize the power flow on the boundaries in Eq. (16). As demonstrated in Fig. 4, the field is generated by the current source, namely, the dipole emitter, which is implemented by a small electric line current. In our model, the dipole is oriented horizontally. For an electric current source with finite size of $l\left(l \ll \lambda_{0}\right)$, and linear distribution of current $I_{0}$, the dipole moment of the source ${ }^{45}$ is, $\mu=j I_{0} l / \omega$. In order to avoid higher order multipole moments, the size of the current source should be restricted below a certain value. Our numerical tests show that the variation of the total power dissipation from the size dependence of the emitter is negligible when the size of the emitter is smaller than $2 \mathrm{~nm}$.

Basically, the accuracy of $\gamma_{\text {total }} / \gamma_{0}$ depends on the length of the plasmonic waveguide, which is studied in Appendix A (Appendix B) for the metallic nanowire (square plasmonic waveguide). Accordingly we estimate the relative error on the computed data of $\gamma_{\text {total }} / \gamma_{0}$ for the metallic nanowire (square plasmonic waveguide) to be $\pm 2.5 \%( \pm 2.0 \%)$ in the following calculations for $L_{0}$ larger than $1 \mu \mathrm{m}$.

\section{RESULT AND DISCUSSION}

Due to the tight confinement of the plasmonic mode as well as the geometric slowing down of the mode propagation, ${ }^{24}$ one can achieve very efficient coupling of a single quantum emitter to metallic nanowires, ${ }^{24}$ which was previously studied mainly by employing a quasistatic approximation. ${ }^{46}$ Here, we quantitatively study the spontaneous emission of an emitter in nanoscale proximity to a plasmonic waveguide beyond the quasistatic approximation, which is necessary when one is concerned with realistic plasmonic waveguides that are compatible with current nanofabrication technology. Normally it is claimed that the quasistatic approximation is valid when the size of the structure and the distance of the emitter to the surface are substantially less than the radiation wavelength. To resolve this ambiguous criterion, we compare our numerics with the results obtained within the quasistatic approximation and give a qualitative assessment regarding the validity of the quasistatic approximation. 
We calculate the full electrodynamic solutions by using the rigid full-vectorial three-dimensional finite-element method, as detailed in Sec. II. Our numerical values and the quasistatic values of the plasmonic decay rate and spontaneous emission $\beta$ factor of the gold nanowire with different radius are plotted in Fig. 5. The radius of the gold nanowire varies from 5 to $100 \mathrm{~nm}$. Figure 5 shows the transition of the quasistatic approximation from approximate to inadequate, depending on the size of the radius with a cross indicating $R=20 \mathrm{~nm}$. According to the deviations between the numerical values and the quasistatic approximated values, the ten subplots associated with different radius in Fig. 5 are grouped into three different regimes, namely, the quasistatic approximation regime, the skin depth regime, and the fullwave regime in which the quasistatic approximation breaks down. The quasistatic approximation regime is limited to radii of the nanowire less than $10 \mathrm{~nm}$, as shown in Figs. 5(a)-5(d), where we observe a good agreement between our numerical values and the quastatic approximated values. In this regime, the radius of the wire is 100 times smaller than the vacuum wavelength, meanwhile the field is strongly localized to an area on the order of the cross section of the gold nanowire, which results in the vanishing of the wave properties in the transverse plane and the applicability of the quasistatic approximation. We also note that in Fig. 5(b) there is a few percent deviation between the FEM-simulated spontaneous emission $\beta$ factor and the quasistatic approximated values due to numerical artifacts, since it is not easy to obtain a good convergence when both the radius of the wire and the distance of the emitter to the wire are very small. For large wires, as indicated in Figs. $5(\mathrm{~g})-5(\mathrm{j})$, we find significantly larger values of $\gamma_{p l} / \gamma_{0}$ and the $\beta$ factor compared to those obtained in the quasistatic approximation. The break down of the quasistatic approximation is due to the incapability of describing the coupling between the electric field and the magnetic field that form the propagating waves, since the quasistatic approximation assumes that the magnetic field vanishes. One may suspect that one can still obtain reasonable results from the quasistatic approximation if the radius of the nanowire is $\frac{1}{20}$ of the vacuum wavelength, namely, $50 \mathrm{~nm}$ in our case, since 20 sampling points in one period normally is good enough to resolve the wave properties. However, our numerical calculations, shown in Figs. $5(\mathrm{~g})$ and $5(\mathrm{~h})$, indicate that this assumption is incorrect. Even for wires as small as $50 \mathrm{~nm}$, the quasistatic approximation breaks down, consistent with Akimov's experimental work. ${ }^{10}$ The full electrodynamic solutions predict significantly larger values, up to 5-10 times, compared with the quasistatic approximation, even though the radius of the nanowire is $\frac{1}{10}$ or $\frac{1}{20}$ of the vacuum wavelength.

Figures 5(e) and 5(f) and others with the size of the radius close to the skin depth are grouped into the skin depth regime. In this regime, the deviation between the full electrodynamic solutions and the quasistatic approximation is somewhere in between, compared with Figs. 5(a)-5(d) and $5(\mathrm{~g})-5(\mathrm{j})$. To further study the influence of the skin depth and the limitations of the quasistatic approximation, we investigate the radius dependence of $\frac{\gamma_{p l, \mathrm{FEM}}}{\gamma_{\text {pl,quasi }}}$, which denotes the ratio of the plasmonic decay rate obtained from our numerical method and that of the quasistatic approximation, for metals with different skin depth, cf. Fig. 6. In Fig. 6, the distance of the emitter to the surface of the metals, i.e., $d$, is a fixed value for each curve. As can be seen from Fig. 6, the deviation between the two results strongly depends on the size of the radius as well as the optical properties of the metals, namely, the skin depth. Coincidentally, the ratio of the plasmonic decay rate obtained from our numerical method and the quasistatic approximation for the four different metals is around 1.5 when the radius of the nanowire equals the skin depth. In other words, one can qualitatively assess the validity of the quasistatic approximation by considering the ratio between the radius of the metallic nanowire and the skin depth. The electric field is severely depleted in the center of the metallic nanowire when the radius is larger than the skin depth, and for the fundamental mode the electric field is mediated by the magnetic field, which reaches its maximum in the depletion area of the electric field. However, in the quasistatic approximation there is no magnetic field to couple with the electric field, which significantly impacts the field distribution and reduces the plasmonic decay rate and the spontaneous emission $\beta$ factor. Based on our numerical calculations, we conclude that the quasistatic approximation is valid only when the radius is smaller than the skin depth of the metals.

To extend the cylindrical wire to other structures, i.e., rectangular metallic waveguide or slot waveguides which are more compatible with current lithographic fabrication technology, we studied the coupling of the quantum emitter with a square plasmonic waveguide as an example. As shown in the inset in Fig. 7, the quantum emitter is oriented along the $X$ axis, and the distance dependence of the plasmonic decay rates and spontaneous emission $\beta$ factors is calculated as function of distance from the emitter to the metal surface along the $X$ axis. For the square plasmonic waveguide, though the electric field of the fundamental mode is concentrated around the four corners, one can achieve an efficient coupling between the plasmonic mode and a horizontally oriented quantum emitter. With optimized side length of the waveguide and distance of the emitter to the edge of the waveguide, the $\beta$ factor can reach $80 \%$.

\section{CONCLUSION}

In conclusion, we developed a self-consistent model to quantitatively study the spontaneous emission of a quantum emitter at nanoscale proximity to a plasmonic waveguide using the finite-element method, which is beyond the quasistatic approximation. The dyadic Green's function of the guided modes supported by the plasmonic waveguide can be constructed numerically from the eigenmode analysis, and subsequently the normalized decay rate into the plasmonic channel can be extracted. The 3D finite-element model is also implemented to calculate the total decay rate, including the radiative decay rate, nonradiative decay rate, and the plasmonic decay rate. In the 3D model, it is assumed that only one guided plasmonic mode is dominatingly excited, which is normally true when the size of the cross section of the plasmonic waveguide is below $100 \mathrm{~nm}$. Under such con- 


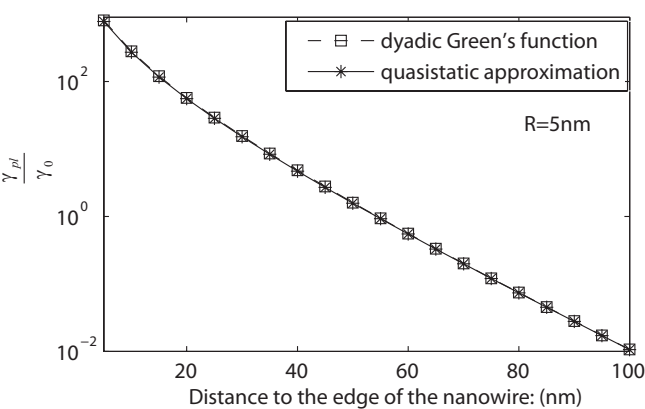

(a)

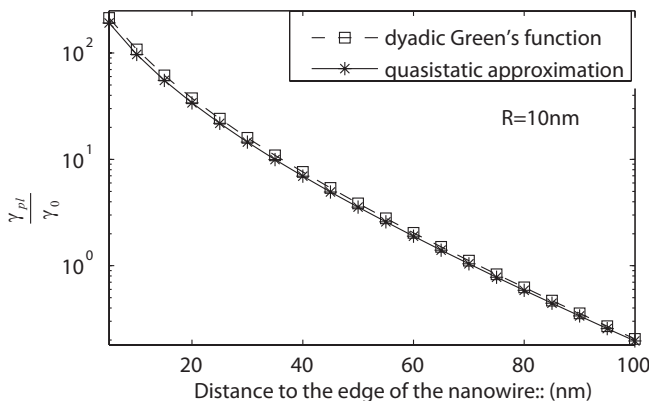

(c)

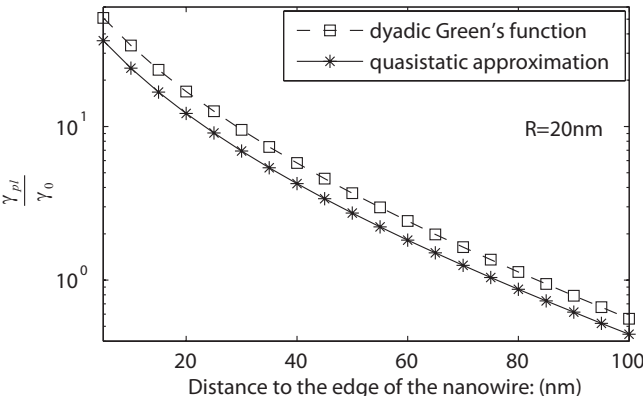

(e)

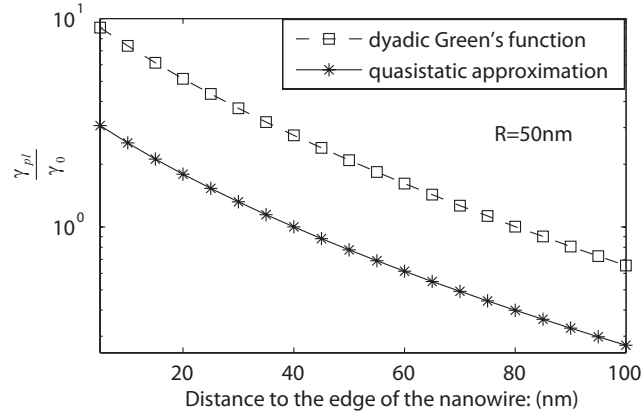

(g)

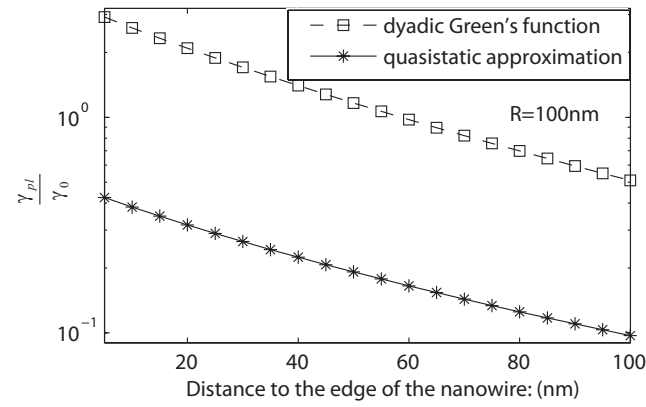

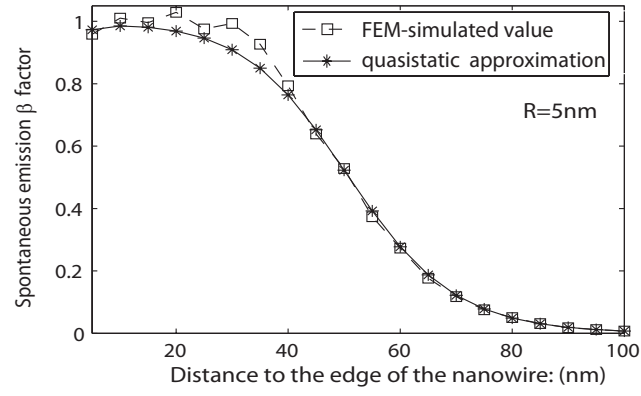

(b)

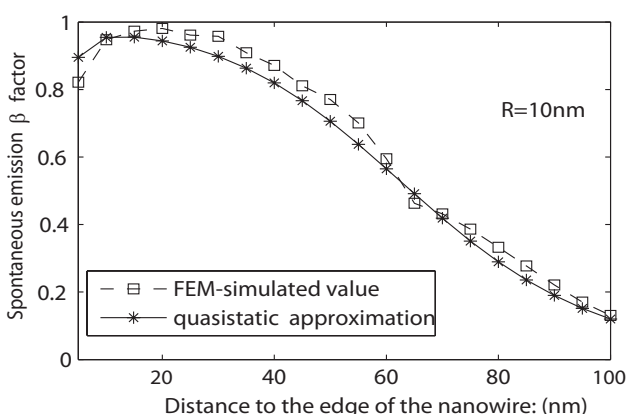

(d)

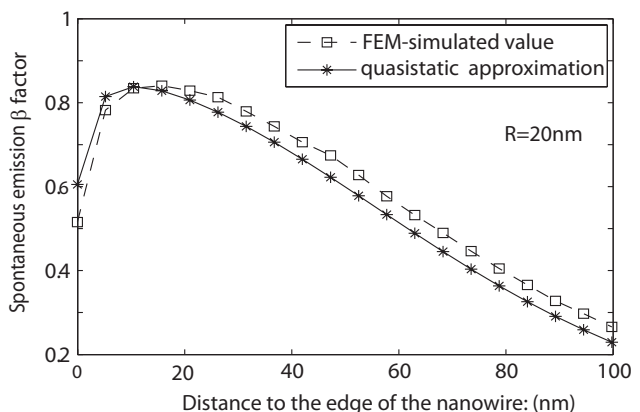

(f)

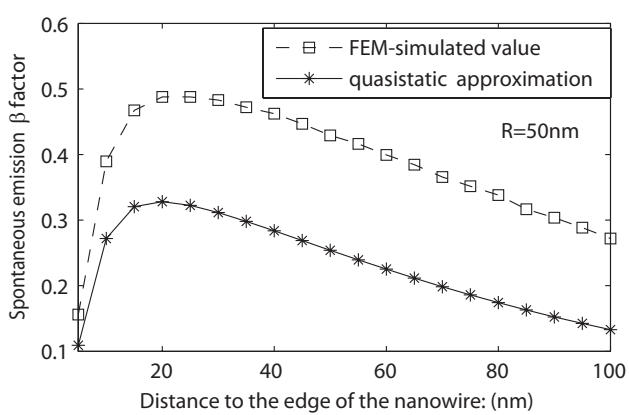

(h)

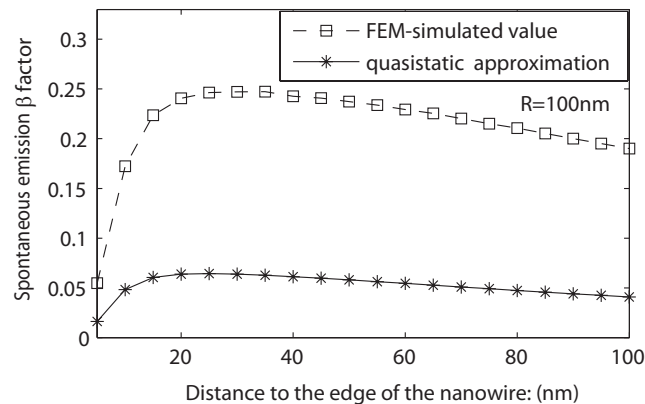

(j)

FIG. 5. Comparison of FEM-simulated results based on the dyadic Green's function with the quasistatic approximation for the metallic nanowire. 


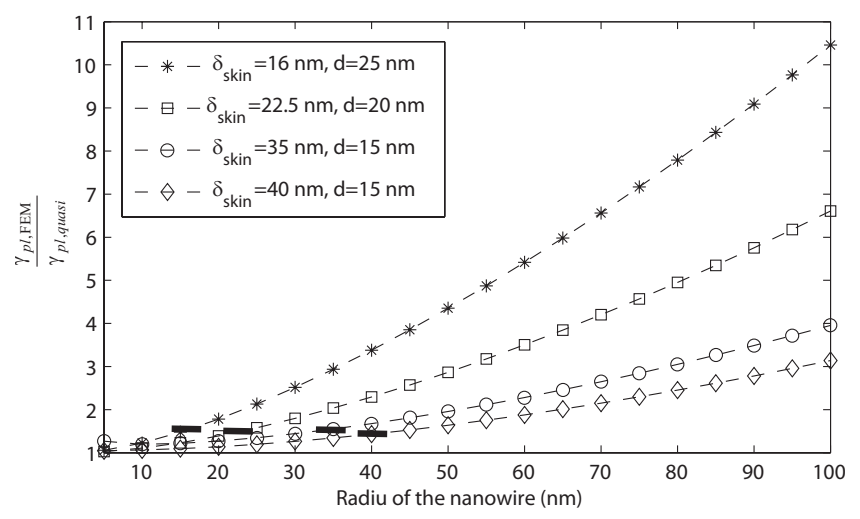

FIG. 6. The radius dependence of $\frac{\gamma_{p l \text {. FEM }}}{\gamma_{\text {pl,quasi }}}$ for four different metals. The skin depths of the four different metals are 16, 22.5, 35, and $40 \mathrm{~nm}$, the corresponding relative optical permittivities are -100 $+3.85 i,-50+3.85 i,-20+3.85 i$, and $-15+3.85 i$. The vacuum wavelength is $1 \mu \mathrm{m}$.

dition, the spontaneous emission $\beta$ factor is calculated. We compared our numerical approach with the quasistatic approximation for the gold nanowire. The comparison shows that our results are 5-10 times larger values for the normalized plasmonic decay rate $\gamma_{p l} / \gamma_{0}$ and the spontaneous emission $\beta$ factor compared to the values obtained in the quasistatic approximation. We examined the limitation of the quasistatic approximation by taking into account the skin depth of the metals. We conclude that the quasistatic approximation is valid only when the radius is smaller than the skin depth of the metals. We also applied our numerical model to calculate the spontaneous emission of a quantum emitter coupled to a square plasmonic waveguide. The numerical calculations show that spontaneous emission $\beta$ factors up to $80 \%$ can be achieved for a horizontal dipole emitter, when the distance and the side length are optimized.

\section{ACKNOWLEDGMENTS}

The authors would like to thank Anders S. Sørensen, Tho-
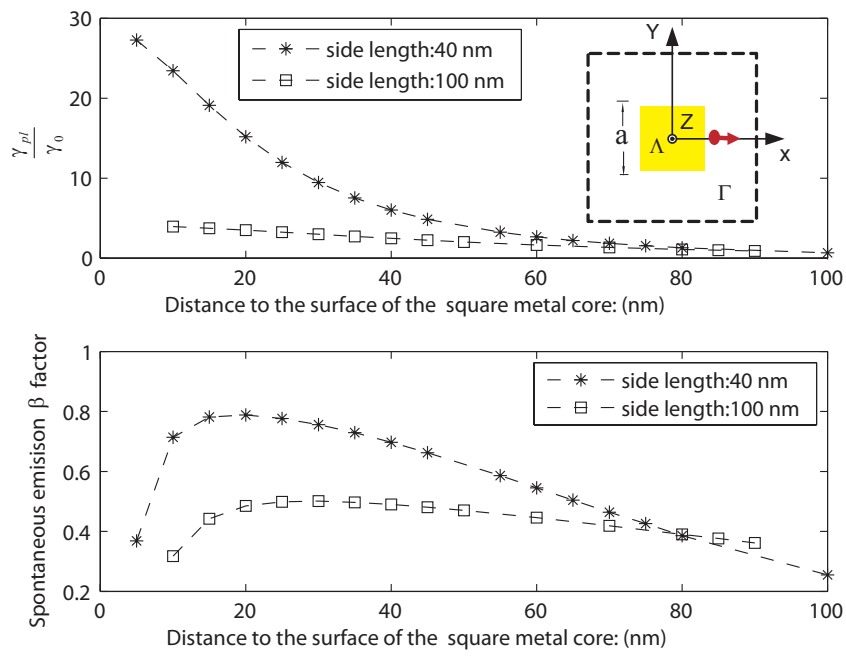

FIG. 7. (Color) Distance dependence of the plasmonic decay rates and spontaneous emission $\beta$ factors for the square plasmonic waveguide.

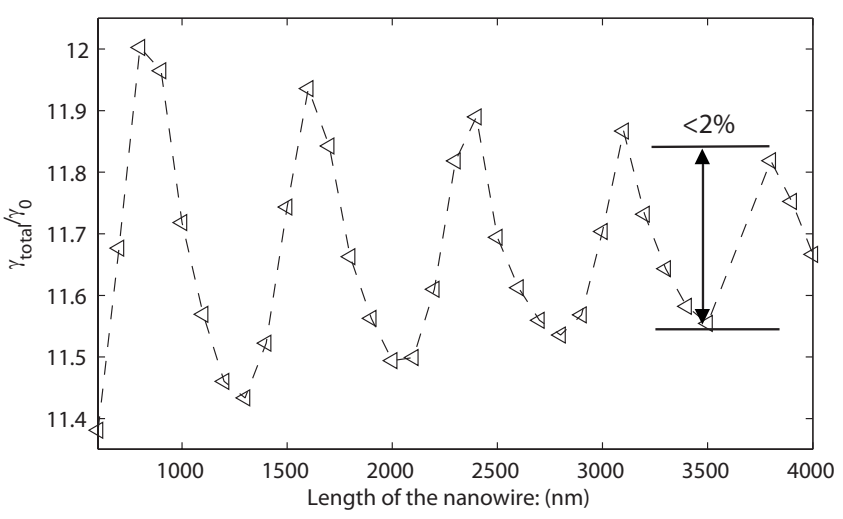

FIG. 8. Length-dependence study of the total decay rate for the metallic nanowire. The radius of the metallic nanowire is $20 \mathrm{~nm}$, the distance of emitter to the wire edge is $30 \mathrm{~nm}$.

mas Søndergaard, Andrei Lavrinenko, and Darrick Chang for fruitful discussions. We further acknowledge Femius Koenderink for careful reading of the manuscript. We gratefully acknowledge support from Villum Fonden.

\section{APPENDIX A: LENGTH DEPENDENCE OF TOTAL DECAY RATE FOR THE METALLIC NANOWIRE}

In order to check the validity of the mode matching boundary condition we studied the length dependence of the total decay rate for two different plasmonic waveguides. The length dependence of the total decay rate $\gamma_{\text {total }}$ for the metallic nanowire is shown in Fig. 8. The fundamental mode supported by the metallic nanowire is TM, hence the mode matching boundary condition defined by Eq. (14a) is implemented. As can be seen from Fig. 8, the variation in the total decay rate is reduced by increasing $L_{0}$, and the damped oscillation of the total decay rate with $L_{0}$ indicates a certain amount of reflection from radiation modes, which is confirmed by the period of the oscillation (equal to the wavelength in a media with $\varepsilon=2$ ). We also see that the variation in the total decay rate due to the length dependence is below $\pm 2.5 \%$ due to the dominating excitation of the plasmonic mode for $L_{0}$ larger than $1.0 \mu \mathrm{m}$. The relative error on the computed data is even smaller, less than $\pm 1.0 \%$ for $L_{0}$ larger than $1.75 \mu \mathrm{m}$.

\section{APPENDIX B: LENGTH DEPENDENCE OF TOTAL DECAY RATE FOR SQUARE PLASMONIC WAVEGUIDE}

Regarding the square plasmonic waveguide, the condition defined by Eq. (15) is applied on the boundary to absorb the hybrid mode supported by the waveguide, where $\bar{H}_{0}$ is the magnetic field for the matched field. As shown in Fig. 9, there is also a damped oscillation of the total decay rate with the length of the computation domain, and the tendency of achieving higher accuracy for $\gamma_{\text {total }}$ when $L_{0}$ is lengthened, which is similar to the length dependence study of the total decay rate for the nanowire. Nevertheless, there are two distinctions between the two plots: (I) the variation in the total decay rate for the square plasmonic waveguide is much larger than that for the metallic nanowire; (II) the variation in 


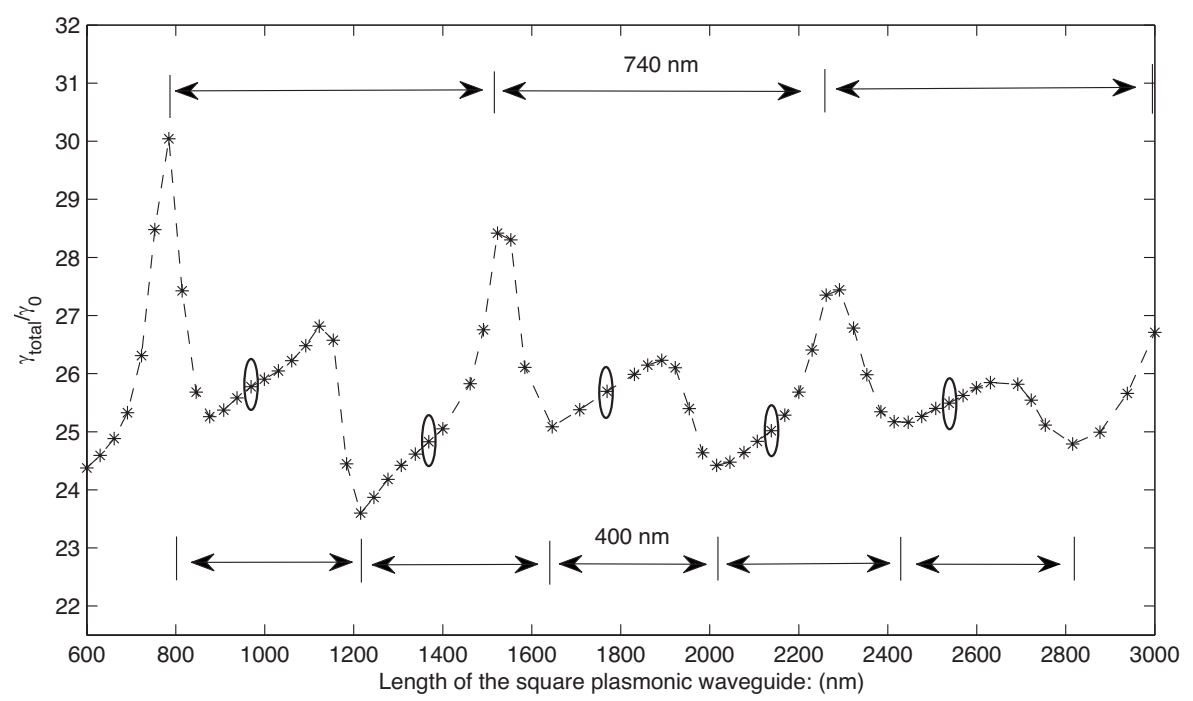

(a)

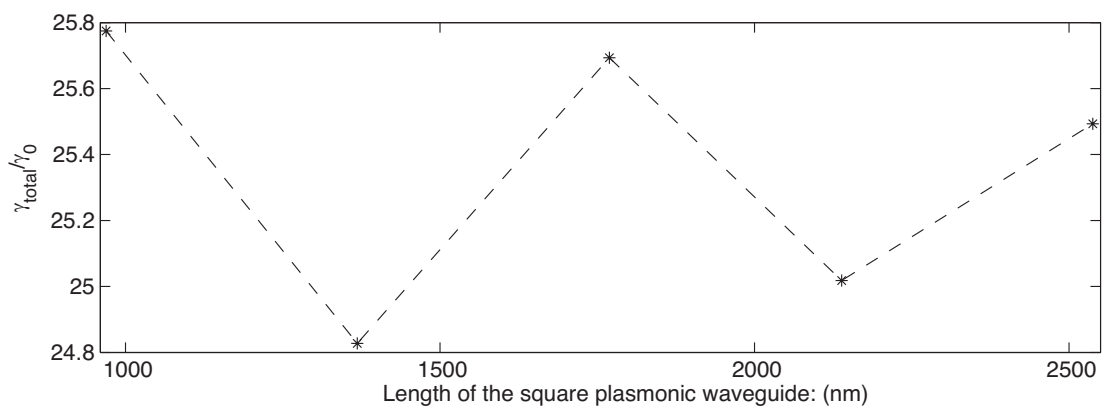

(b)

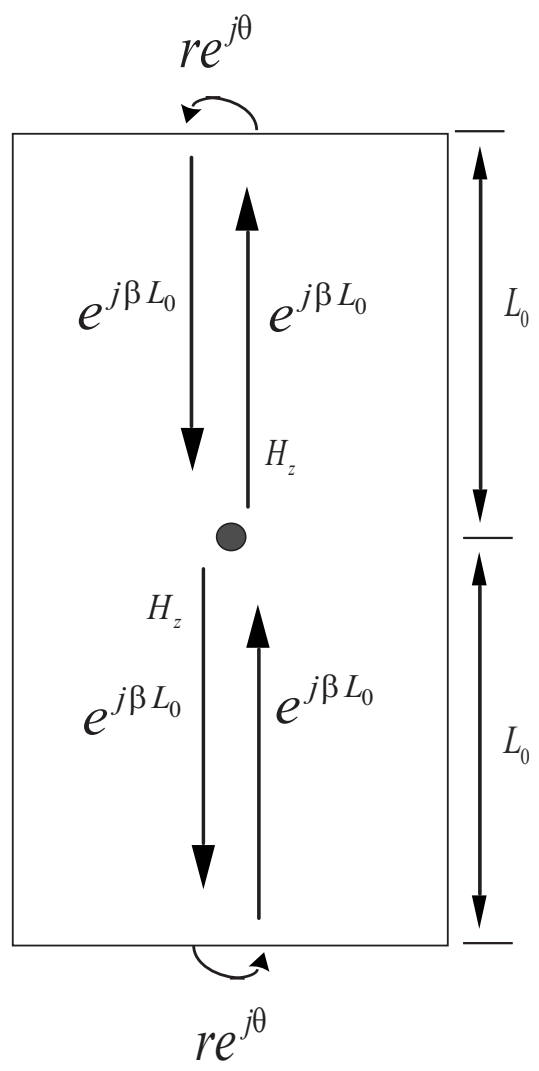

(c)

FIG. 9. (a) Length dependence of the total decay rate for the square plasmonic waveguide. The side length is $30 \mathrm{~nm}$, the distance of the emitter to the edge of the square metal core is $20 \mathrm{~nm}$. (b) Length dependence for the points from (a) (marked by ellipses) where $\operatorname{real}\left(e^{j\left(\beta L_{0}-\phi\right)}\right)=0$ holds approximately. (c) Illustration of the reflection of the normal magnetic field of the fundamental hybrid in the $3 \mathrm{D}$ model, with $r$ and $\theta$ being the reflection coefficient and phase shift, respectively.

the total decay rate for the square plasmonic waveguide with $L_{0}$ primarily stems from the reflection of two different modes, which are indicated by two different periods in the damped oscillation. The reflection of the fundamental mode, which is supposed to be absorbed at the boundaries, is responsible for the oscillation with the period of $400 \mathrm{~nm}$, the other oscillation with the period of $740 \mathrm{~nm}$ results from the reflection of a quasiguided mode, denoted by $E_{q g}$. The explanation is the following: the boundary condition defined by Eq. (14a) can completely absorb the matched pure TM mode, while it is not true for the boundary condition defined by Eq. (15) for the hybrid mode, and a significant reflection from a quasiguided mode also exists for the square plasmonic waveguide. For the hybrid mode the last term in Eq. (13) relies not only on the tangential components of the electric (magnetic) field but also on the normal component of the electric (magnetic) field, which is intrinsically lost on the boundary in the vector-element formulation of the $3 \mathrm{D}$ numerical model. ${ }^{43}$ Our interpretation is that even though the normal component of the electric field can be included on the boundaries by Eq. (15), the normal component of the magnetic field is essentially missing in our 3D FEM model that employs a vector finite-element formulation, ${ }^{47,48}$ resulting in the reflections in the total decay calculations for the square plas- monic waveguide. However, in Fig. 9(a), it appears that the points for which $\operatorname{real}\left(e^{j\left(\beta L_{0}-\phi\right)}\right)=0$ holds approximately converge quickly with minimum impact of the reflection from the fundamental hybrid mode. The mode $E_{q g}$, with effective wavelength of $740.07 \mathrm{~nm}$, is characterized by the material properties of the waveguide and is rather insensitive to the size of the metallic core. Compared with other quasiguided modes or radiation modes, the mode $E_{q g}$ has a relatively significant contribution to $\gamma_{\text {total }}$, the normalized spontaneous emission rate is 0.107 . Since no extra effort is made to prevent the reflections of other components of the mode $E_{q g}$, it is understandable that the induced reflections give rise to several peaks in Fig. 9(a).

The normal component of the magnetic field of the fundamental mode in the 3D model can be obtained by a $2 \mathrm{D}$ eigenvalue calculation, $H_{n, l}=\sqrt{\frac{\gamma_{p l} P_{0}}{\gamma_{0} P_{2 d}}} H_{2 d}^{n} e^{j(\beta l-\phi)}$, where $l$ is the distance from the observation plane to the emitter. Similarly, the reflected normal component of the magnetic field at the position of the emitter can be obtained by taking into account the phase shift due to propagation and reflection, $H_{n, 0}^{r}$ $=r \sqrt{\frac{\gamma_{p l} P_{0}}{\gamma_{0} P_{2 d}}} H_{2 d}^{n} e^{j\left(2 \beta L_{0}-\phi+\theta\right)}$, as shown in Fig. 9(c). The reflected normal component of the magnetic field will "generate" a perturbation term $E_{x}^{r}$ to the original $E_{x}$ component, the real 
part of which is integrated to calculate the total power dissipation. According to Table I, the reflected term $E_{x}^{r}$ from the fundamental hybrid mode is given by

$$
E_{x}^{r}=-\frac{1}{\omega \varepsilon_{0} \varepsilon(\bar{r})}\left[\nabla_{t} \times \bar{n}\left(r \sqrt{\frac{\gamma_{p l} P_{0}}{\gamma_{0} P_{2 d}}} H_{2 d, m}^{z} e^{j\left(2 \beta L_{0}-\phi+\theta\right)}\right)\right]_{x} .
$$

The real part of $E_{x}^{r}$ can be zero when $L_{0}$ is appropriately chosen, therefore, the obtained total decay rates are expected to approach the true values more closely due to the vanishing contribution of $E_{x}^{r}$ to the total decay rate. In Fig. 9(a), at the points with marked ellipses, the half model length $L_{0}$ fits the requirement $\left[\operatorname{real}\left(E_{x}^{r}\right)=0\right]$, and we also found that the phase shift $\theta$ is required approximately to be $\pi / 2$. Further investigation of the phase shift $\theta$ involves technical details regarding the implementation of the vector-element formulation of the finite-element method, which is beyond the scope of the present paper, we refer to the Refs. 43 and 47-49. *yche@fotonik.dtu.dk

${ }^{1}$ E. M. Purcell, Phys. Rev. 69, 681 (1946).

${ }^{2}$ H. P. Urbach and G. L. J. A. R. Rikken, Phys. Rev. A 57, 3913 (1998).

${ }^{3}$ J. Johansen, S. Stobbe, I. S. Nikolaev, T. Lund-Hansen, P. T. Kristensen, J. M. Hvam, W. L. Vos, and P. Lodahl, Phys. Rev. B 77, 073303 (2008)

${ }^{4}$ G. Björk, S. Machida, Y. Yamamoto, and K. Igeta, Phys. Rev. A 44, 669 (1991).

${ }^{5}$ J. M. Gérard, B. Sermage, B. Gayral, B. Legrand, E. Costard, and V. Thierry-Mieg, Phys. Rev. Lett. 81, 1110 (1998).

${ }^{6}$ E. Yablonovitch, Phys. Rev. Lett. 58, 2059 (1987).

${ }^{7}$ P. Lodahl, A. F. van Driel, I. S. Nikolaev, A. Irman, K. Overgaag, D. Vanmaekelbergh, and W. L. Vos, Nature (London) 430, 654 (2004).

${ }^{8}$ D. Kleppner, Phys. Rev. Lett. 47, 233 (1981).

${ }^{9}$ T. Lund-Hansen, S. Stobbe, B. Julsgaard, H. Thyrrestrup, T. Sünner, M. Kamp, A. Forchel, and P. Lodahl, Phys. Rev. Lett. 101, 113903 (2008).

${ }^{10}$ A. V. Akimov, A. Mukherjee, C. L. Yu, D. E. Chang, A. S. Zibrov, P. R. Hemmer, H. Park, and M. D. Lukin, Nature (London) 450, 402 (2007).

${ }^{11}$ D. E. Chang, A. S. Sørensen, P. R. Hemmer, and M. D. Lukin, Phys. Rev. Lett. 97, 053002 (2006).

${ }^{12}$ K. Kneipp, Y. Wang, H. Kneipp, L. T. Perelman, I. Itzkan, R. R. Dasari, and M. S. Feld, Phys. Rev. Lett. 78, 1667 (1997).

${ }^{13}$ S. Nie and S. R. Emory, Science 275, 1102 (1997).

${ }^{14}$ N. E. Hecker, R. A. Hopfel, N. Sawaki, T. Maier, and G. Strasser, Appl. Phys. Lett. 75, 1577 (1999).

${ }^{15}$ K. B. Crozier, A. Sundaramurthy, G. S. Kino, and C. F. Quate, J. Appl. Phys. 94, 4632 (2003).

${ }^{16}$ T. H. Taminiau, F. D. Stefani, F. B. Segerink, and N. F. van Hulst, Nat. Photonics 2, 234 (2008).

${ }^{17}$ S. Kühn, U. Hakanson, L. Rogobete, and V. Sandoghdar, Phys. Rev. Lett. 97, 017402 (2006).

${ }^{18}$ D. Chang, A. Sørensen, E. Demler, and M. Lukin, Nat. Phys. 3, 807 (2007).

${ }^{19}$ W. L. Barnes, A. Dereux, and T. W. Ebbesen, Nature (London) 424, 824 (2003).

${ }^{20}$ A. V. Zayats, J. Elliott, I. I. Smolyaninov, and C. C. Davis, Appl. Phys. Lett. 86, 151114 (2005).

${ }^{21}$ I. I. Smolyaninov, J. Elliott, A. V. Zayats, and C. C. Davis, Phys. Rev. Lett. 94, 057401 (2005).

${ }^{22}$ J. Takahara, S. Yamagishi, H. Taki, A. Morimoto, and T. Kobayashi, Opt. Lett. 22, 475 (1997).
${ }^{23}$ S. I. Bozhevolnyi, V. S. Volkov, E. Devaux, J.-Y. Laluet, and T. W. Ebbesen, Nature (London) 440, 508 (2006).

${ }^{24}$ D. E. Chang, A. S. Sørensen, P. R. Hemmer, and M. D. Lukin, Phys. Rev. B 76, 035420 (2007).

${ }^{25} \mathrm{G}$. Veronis and S. Fan, Opt. Lett. 30, 3359 (2005).

${ }^{26}$ Y. C. Jun, R. D. Kekapture, J. S. White, and M. L. Brongersma, Phys. Rev. B 78, 153111 (2008).

${ }^{27}$ E. D. Palik, Handbook of Optical Constants of Solids II (Academic Press, New York, 1991).

${ }^{28}$ J. Jung, T. Søndergaard, and S. I. Bozhevolnyi, Phys. Rev. B 76, 035434 (2007).

${ }^{29}$ P. Berini, Phys. Rev. B 61, 10484 (2000).

${ }^{30}$ S. J. Al-Bader, IEEE J. Quantum Electron. 40, 325 (2004).

${ }^{31}$ A. Degiron and D. Smith, Opt. Express 14, 1611 (2006).

${ }^{32}$ S. Chattopadhyay and P. K. Saha, J. Opt. A, Pure Appl. Opt. 10, 095201 (2008).

${ }^{33}$ T. Søndergaard and B. Tromborg, Phys. Rev. A 64, 033812 (2001).

${ }^{34}$ L. Novotny and B. Hecht, Principles of Nano Optics, 2nd ed. (Cambridge University Press, Cambridge, 2006).

${ }^{35}$ A. W. Snyder and J. Love, Optical Waveguide Theory (Springer, New York, 1983).

${ }^{36}$ C. F. Bohren and D. R. Huffman, Absorption and Scattering of Light by Small Particles (Wiley, New York, 1983).

${ }^{37}$ J. A. Stratton, Electromagnetic Theory, 1st ed. (McGraw-Hill, New York, 1941).

${ }^{38}$ A. F. Koenderink, M. Kafesaki, C. M. Soukoulis, and V. Sandoghdar, J. Opt. Soc. Am. B 23, 1196 (2006).

${ }^{39}$ Y. Xu, R. K. Lee, and A. Yariv, Phys. Rev. A 61, 033807 (2000).

${ }^{40}$ C. Hermann and O. Hess, J. Opt. Soc. Am. B 19, 3013 (2002).

${ }^{41}$ J.-C. Weeber, A. Dereux, C. Girard, J. R. Krenn, and J.-P. Goudonnet, Phys. Rev. B 60, 9061 (1999).

${ }^{42}$ B. T. Draine and P. J. Flatau, J. Opt. Soc. Am. A 11, 1491 (1994).

${ }^{43}$ J. M. Jin, The Finite Element Method in Electromagnetics, 2nd ed. (Wiley, New York, 2002).

${ }^{44} \mathrm{http}: / / \mathrm{www} . \mathrm{comsol} . \mathrm{com}$

45 J. D. Jackson, Classical Electrodynamics, 3rd ed. (Wiley, New York, 1999).

${ }^{46}$ V. V. Klimov and M. Ducloy, Phys. Rev. A 69, 013812 (2004).

${ }^{47}$ V. N. Kanellopoulos and J. P. Webb, IEEE Trans. Microwave Theory Tech. 43, 2168 (1995).

${ }^{48}$ J. P. Webb, IEEE Trans. Magn. 29, 1460 (1993).

${ }^{49}$ M. M. Botha and D. B. Davidson, IEEE Trans. Antennas Propag. 54, 3499 (2006). 